\title{
Ideas alternativas sobre el Trabajo Fin de Grado en estudiantes de Magisterio
}

José Manuel Pérez Martín. Universidad Autónoma de Madrid

Sergio Martínez Luna. Universidad Carlos III de Madrid /Universidad Camilo José Cela

Recepción: 24 de octubre de 2017 | Aceptado: 10 noviembre de 2017

Correspondencia: José Manuel Pérez | Correo-e: josemanuel.perez@uam.es

\author{
iD http:// orcid.org/0000-0002-0658-9050 \\ Citar: Pérez-Martín, JM. y Martínez-Luna, S. (2017). Ideas alternativas sobre el Trabajo Fin de \\ Grado en estudiantes de Magisterio. ReiDoCrea, 6, 246-259.
}

\begin{abstract}
Resumen: La normativa actual recoge la necesidad de formar a los futuros maestros en el ámbito de la investigación y la innovación educativas, por lo que se ha incluido la asignatura del Trabajo de Fin de Grado (TFG) en los planes de estudio. Sin embargo, les supone grandes dificultades superarla. Objetivo: Este artículo analiza las dificultades a las que se enfrentan los estudiantes de los Grados de Educación Infantil y Primaria a la hora de elaborar el TFG. Método: Para detectar las ideas alternativas que surgen durante la elaboración de estos trabajos, hemos analizado 43 TFG de los grados de Educación Infantil y Primaria. Resultados: De entre los resultados obtenidos, destacan como principales errores, las dificultades en la gestión de la bibliografía y la justificación y representación de sus datos. Conclusiones: Por último, se plantean algunas propuestas de mejora con el fin de que esta asignatura resulte productiva para el desarrollo de las competencias investigadoras de los estudiantes de educación. Entre ellas hay que destacar la inclusión de actividades evaluables en otras asignaturas de los grados en las que se manejase información de la misma forma en la que se exige en los TFG.
\end{abstract}

Palabras clave: Formación de profesores | Trabajo de investigación

\section{Alternative conceptions about undergraduate dissertations in Education students}

\begin{abstract}
The current regulations include the need to train future teachers in the field of educational research and innovation; which is the reason why the undergraduate dissertation (UD) has been included in the curriculum. However, pre-service teachers often have great difficulty passing it. Aim: This article analyzes the difficulties faced by students taking Degrees in Pre-school and Primary Education when preparing the UD. Method: In order to detect the alternative ideas that arise during the preparation of these research assignments, we analyzed a sample of 43 undergraduate dissertations of the Pre-school and Primary Education degrees. Results: Among the results obtained, the main errors are difficulties in the management of the bibliography and the justification and representation of the data. Conclusions: Finally, we proposed some suggestions for improvement in order to make this course useful for the development of research skills in students of education degrees. Among them, we must point out the inclusion of training activities in other courses of the education degrees in which information and bibliography are used in the same way as in the UD.
\end{abstract}

Keywords: Teacher training | Research study

\section{Introducción}

En los últimos años se ha cuestionado de la necesidad de cambios educativos en España. La Declaración de Bolonia (1999) o Espacio Europeo de Educación Superior (EEES) ha sido la reforma educativa de mayor calado realizada en nuestro país, ya que afecta a la profesionalización. En ella se plantea que los titulados universitarios tienen que adquirir e integrar destrezas profesionales al finalizar su plan de estudios. Para conseguir este objetivo, las transposiciones legislativas acometidas, RD 1393/2007 sobre las enseñanzas universitarias oficiales, y la posterior modificación por parte del RD 861/2010, determinan que los planes de estudios oficiales de todas las titulaciones universitarias de grado finalicen con una asignatura obligatoria en la que se elabore y defienda un Trabajo de Fin de Grado. 
Los trabajos de fin de estudios no eran frecuentes en las universidades españolas y para su creación se partía de cero en la mayoría de las facultades (Mateo, 2009; Álvarez y Pascual., 2012; Bonilla, Fuentes, Vacas \& Vacas, 2012), ya que eran generalmente exclusivos de titulaciones científico-técnicas (Arteaga, Ahedo, Gutiérrez GómezCalcerrada y Solera, 2013; Hernández-Leo et al., 2013), por lo que ha resultado todo un desafío su puesta en marcha en Facultades de Ciencias Sociales, que carecían de tradición en este tipo de acciones formativas. En cambio, la experiencia en Europa con esta asignatura es amplia (Webster, Pepper y Jenkins, 2000; Neave, 2003; Rowley y Slack, 2004; Reynolds y Thompson, 2011). Sin embargo, la concreción legislativa española que define qué es un TFG, su organización y desarrollo no está clara (Rullán, Estapé-Dubreuil, Fernández y Márquez, 2010; Rekalde Rodríguez, 2011; Álvarez y Pascual, 2012). En ella se dice que es un trabajo autónomo, personal y original que permite a los estudiantes, bajo la orientación de un tutor, articular de forma coherente e integrada los contenidos formativos recibidos a lo largo del grado, con el fin de demostrar las competencias adquiridas asociadas al título (MEC, 2007; 2010).

Por ello, las comisiones que elaboraron los planes de estudio del EEES, lo enfocaron como un trabajo que pretende la integración de las competencias adquiridas a lo largo de los estudios de grado (Rullán et al., 2010; Valderrama et al., 2010). No obstante, los análisis llevados a cabo sobre las guías docentes coinciden en que se trata de una asignatura muy heterogénea en diseño y evaluación (Rekalde Rodríguez, 2011; Hernández-Leo et al., 2013; López-Cózar, Priede y Benito Hernández, 2013).

En España, el Proyecto Tuning (2009) se tomó como punto de partida para fijar las directrices sobre la evaluación de competencias en el EEES, pero siempre con pequeñas modificaciones en su redacción, que permitieran marcos de evaluación particulares debido a la idiosincrasia de cada grado (Rullán et al., 2010). De modo que podemos decir que existe consenso, aunque no literalidad, en ciertas competencias de tipo transversal que deben tener los titulados universitarios al finalizar sus estudios, como es el caso de la comunicación oral y escrita en lengua nativa; la habilidad de búsqueda y gestión de la información; la capacidad de organizar y planificar; la capacidad de análisis y síntesis; y la capacidad para resolver problemas (Rullán et al., 2010; Valderrama et al., 2010; Álvarez y Pascual, 2012; Bonilla et al., 2012).

Con el fin de cumplir con estos objetivos se ha generalizado el uso de rúbricas en las asignaturas de TFG por ser un modelo que facilita la evaluación (Valderrama et al., 2010; Hernández-Leo et al., 2013), objetivándola y permitiendo la autoevaluación y el aprendizaje mediante la descripción de los indicadores de logro (Rullán et al., 2010). Con todo, el debate se amplió en múltiples frentes como evaluaciones temporalizadas, que no sólo se centran en el producto (el TFG final), sino en el propio desarrollo del trabajo, con el argumento de que las competencias deben ser valoradas mediante una evaluación continua y formativa en diferentes etapas (Rullán et al., 2010; Valderrama et al., 2010; Hernández-Leo et al., 2013); e incluso en cuanto a si la evaluación debe hacerla el tutor, un tribunal (con o sin miembros externos) o entre ambos (Rullán et al., 2010; Hernández-Leo et al., 2013). Estos debates siguen abiertos y no parece que vayan a resolverse próximamente, ya que estudios muy extensos donde se consulta a los agentes implicados arrojan opiniones diversas.

Las competencias mencionadas anteriormente enmarcan al TFG de las distintas titulaciones en el ámbito de la investigación. En cualquier caso, como decíamos anteriormente, no todos los grados dan el mismo peso a este objetivo como parte de su programa formativo. Tanto es así que algunas facultades no permiten a sus alumnos realizar trabajos de investigación (Garrote, 2015). El caso contrario se presenta en las Facultades de Educación, donde es una de las modalidades ofrecidas. Sin embargo, los 
alumnos no tienen clara la implicación de la investigación educativa en el aula (Arteaga et al., 2013) y por ende el papel del TFG en su formación (Cano Vela y Ortiz Ballesteros, 2013). Esta percepción debe cambiar, ya que el marco legislativo otorga la función de innovación a los docentes en su propia aula a través de la investigación educativa, para que sean capaces de desarrollar competencias ligadas a la investigación (Muñoz Cantero y Mato Vásquez, 2014).

En los últimos años ha habido un gran interés por el análisis de resultados de la asignatura de TFG en las diferentes titulaciones, lo que ha generado un importante volumen de estudios publicados en jornadas, libros y revistas (Vera y Briones, 2016), pero fundamentalmente centrados en aspectos metodológicos y de diseño. Cabe señalar que a pesar de ello son minoritarios los estudios que se centran en las debilidades concretas que presentan los alumnos de los Grados de Educación Infantil y Primaria durante el desarrollo de la asignatura.

Muchas de las debilidades que encontramos se deben a las concepciones erróneas que tienen sobre él. Por ello resulta de interés analizar cuáles son concretamente las ideas alternativas, como: qué entienden por título, cómo debe ser, qué partes debe tener el escrito, qué debe contener cada una de ellas, qué se pone en las referencias, en qué formato, qué es citar en el texto, el formato de las figuras, tablas y anexos, su imbricación con el texto, entre otras. De esta manera los tutores pueden intervenir sobre ellas de forma apriorística en las reuniones de seguimiento y mejorar el rendimiento académico de éstos en futuras promociones.

\section{Objetivos}

Presentamos un análisis cuantitativo concreto sobre las debilidades más comunes que aparecen en los TFG de Educación Infantil y Primaria en la Universidad Camilo José Cela (UCJC). En base a esos resultados se proponen unas líneas de actuación para superar tales dificultades. Con ello se pretende que los tutores de TFG y sus coordinadores dispongan de datos para mejorar la asignatura para que ésta represente para los maestros en formación una oportunidad real para conocer y ensayar las competencias investigadoras que se espera que ejerzan en su desempeño profesional.

Este objetivo general se puede descomponer en tres objetivos concretos:

- Detectar las concepciones erróneas sobre el TFG que tienen los alumnos de magisterio.

- Analizar y clasificar los tipos de concepciones erróneas que aparecen en los borradores completos de TFG de los estudiantes de magisterio.

- Elaborar propuestas de intervención para la mejora de la formación previa a la elaboración del TFG.

\section{Método}

\section{Población y Muestra}

Para realizar el estudio, analizamos el primer documento borrador completo que entregaron a sus directores 43 estudiantes de los Grados de Educación Infantil y Primaria de la UCJC en los cursos 2014-15 y 2015-16. 


\section{Instrumento, procedimiento de recogida y análisis de datos}

Como se indica en la introducción, el proceso de tutorización del TFG es diverso, pero debería tener una secuencia definida. En la UCJC no existe obligación de realizarlo en este formato, pero la práctica totalidad de los profesores siguen el avance de los trabajos de sus alumnos a través de sucesivas tutorías, presenciales y/o virtuales. Los datos del estudio se obtuvieron en una fase de ese seguimiento, concretamente se analizó la primera versión completa que los tutores pedían a sus alumnos de TFG ( $n=43$ alumnos). Empleando una hoja de registro con 35 indicadores que se recogen en las tablas 1 a 4, los tutores marcaron sí o no para cada uno de ellos en cada caso. Estos indicadores son ítems concretos de la elaboración del trabajo que están en consonancia con las rúbricas de evaluación que se proponen por Valderrama et al. (2010) y Hernández-Leo et al. (2013). Así se recogieron datos que se representaron como frecuencias para cada una de los apartados, y se agruparon en variables de niveles superiores, denominadas bloques. Además de estas cuestiones se incorporaron dos datos más a cada caso: género y titulación. Todo ello fue procesado, analizado y representado con Microsoft Excel 2013 (EE.UU.).

\section{Resultados}

El análisis sociológico de la muestra indicó que el género (Figura 1A) de la mayoría de los futuros maestros cuyos borradores de TFG fueron estudiados en este trabajo son mujeres $(78,6 \%)$. Asimismo, la gran mayoría de ellos fueron elaborados para la obtención del Grado en Educación Primaria $(71,4 \%)$ y algo más de una cuarta parte para el Grado en Educación Infantil (Figura 1B).

Figura 1. Diagramas de sectores que representan dos variables del perfil sociológico de la muestra: género $(A)$ y titulación $(B)$.

A Género

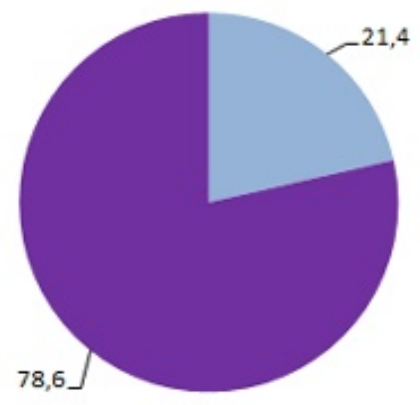

B Titulación

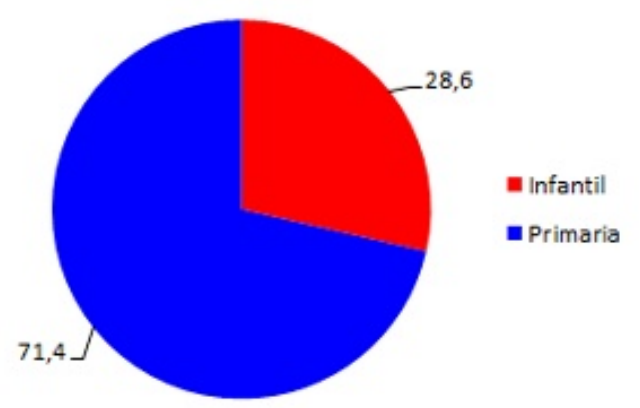

Los resultados de este estudio proceden de las respuestas obtenidas para los 35 puntos de análisis, agrupados en los cuatro bloques con subapartados que se muestran en las tablas 1 a 4.

El bloque I valoraba el conocimiento que tienen los alumnos de TFG sobre la metodología de la investigación. Éste a su vez se desglosó en otros cuatro subapartados: la estructura y organización de los trabajos, el planteamiento de hipótesis, presentación de recursos de apoyo en la presentación de los resultados y la elaboración de la prospectiva. El bloque II analizó los descriptores del trabajo con tres subapartados: 
título, palabras clave y resumen. En el bloque III se valoró el tipo de redacción empleado, desglosándose en cuatro puntos: contenido, estilo, argumentación y legislación. Por último, el bloque IV se centró en las fuentes documentales, compuesto por tres grandes subapartados: formato APA, referencias y citas.

En lo que respecta al subapartado 1 del bloque I, el concepto inicial de los alumnos universitarios de educación sobre la estructura y organización que deben tener los TFG, se analizó en base a cinco ítems (Tabla 1). En este bloque se recoge si existía confusión entre modalidades de trabajos (revisión, investigación e innovación educativa), así como con la organización interna de dichos trabajos de acuerdo con la modalidad indicada.

La primera información que podemos extraer es que los alumnos de los grados de educación de la UCJC mostraron un mayor interés por realizar trabajos de innovación educativa $(39,5 \%)$, seguidos de TFG de investigación educativa $(37,2 \%)$ y por último de revisión teórica $(23,3 \%)$. A pesar de ello, los resultados muestran que aproximadamente 4 de cada 10 alumnos no saben en qué consisten exactamente estas tipologías al entregar este primer borrador completo del trabajo. Tanto es así que encontramos que una elevada frecuencia de alumnos $(34,9 \%)$ consideraba que un TFG debía tener un formato similar a una programación didáctica. De hecho, en muchos casos $(44,2 \%)$ detectamos que entendían que este trabajo consistía en una adaptación de un proyecto de aula o una unidad didáctica. Con estos datos, parece razonable que el 60,5\% de futuros maestros de la muestra presentasen, como objetivos del TFG, los objetivos curriculares de los alumnos de Educación Infantil y Primaria. Este error es de los más frecuentes detectados en nuestro estudio, y demuestra que la idea que tienen los maestros sobre el concepto "objetivo" es fuertemente dependiente de la legislación educativa y su currículum.

El segundo apartado del bloque I analizó la forma en que los alumnos facilitaban el entendimiento de la información aportada en el trabajo (Tabla 1). Para ello estudiamos los recursos de apoyo empleados y su integración con la línea discursiva del texto presentado. Valoramos si se mencionaban las ilustraciones (esquemas, mapas conceptuales, gráficas, tablas, etc.) en el texto, y si el pie de figura y/o tabla recogía mínimamente su contenido y su fuente. Según nuestros resultados, el $58,1 \%$ no presentaba la ilustración con el correspondiente pie. Además 7 de cada 10 maestros en formación no explicaban la información contenida en los recursos de apoyo o no mencionan la fuente de la que proceden. Además, el $65,1 \%$ de los trabajos analizados no se indica en qué momento de la lectura del texto se debe hacer uso de la figura, ni de qué forma hacerlo, con lo que se pone de manifiesto la falta de conexión entre los distintos componentes del documento (texto e ilustraciones). Con todo, no se establecen relaciones entre el discurso redactado y los recursos de apoyo empleados.

Otra forma de utilizar recursos en la presentación de resultados es a través de los anexos. Debido a la limitación de páginas descrita en la guía docente, algunos contenidos pueden presentarse como anexos. No todo el grupo de estudio los utilizó en este primer borrador entregado. Sin embargo, el $72,1 \%$ de los trabajos analizados contenían dicho apartado. De ellos el $25,8 \%$ los presentaban sin una mínima descripción similar a la del pie de figura, y casi uno de cada tres $(29,0 \%)$ no mencionaba en el texto dicho material suplementario. Los anexos son un recurso adecuado para presentar información de interés a los lectores con menos experiencia en el tema sin provocar tedio en los lectores expertos, pero nuestro análisis muestra que algunos alumnos $(16,1 \%)$ los presentaban en el documento de forma injustificada, como si fuese un apartado de obligada aparición en el documento. 
Un punto también relevante para el análisis de la metodología de la investigación en el TFG de maestros fue la formulación de hipótesis (Tabla 1). El primer ítem valoraba si la hipótesis estaba formulada de forma interrogativa o en infinitivo. Ambos hechos resultan muy frecuentes $(23,3 \%)$, ya que algunos maestros en formación entienden que la hipótesis es el objetivo (verbo en infinitivo) o la pregunta de la investigación (interrogativa) y no, respuestas posibles a las preguntas de investigación. Por otro lado, se analizó si estando bien formulada la hipótesis (oración enunciativa afirmativa), el diseño experimental no permitía ser contrastada $(18,6 \%)$.

El último subapartado del bloque I contenía una cuestión sobre la prospectiva (Tabla 1). Todo estudio ha de incluir una breve alusión a las futuras propuestas que deriven de éste. Los maestros en formación deben entender que cualquier investigación responde al menos parcialmente a las preguntas formuladas, pero también que durante el camino recorrido aparecen otras nuevas. Éstas deben ser recogidas en el apartado final de la prospectiva, como fruto de la realización del trabajo y punto de partida para nuevos trabajos de investigación. Sin embargo, este aspecto no fue reflejado en el $27,9 \%$ de los casos.

El segundo bloque de ítems se refiere a los descriptores del trabajo (Tabla 2). Un trabajo resulta inicialmente atractivo si despierta el interés del lector, fundamentalmente mediante el uso de títulos representativos, concretos y sugerentes. A pesar de esta idea, evaluamos trabajos cuyo título no describía su contenido por falta de concreción, no se precisaba la etapa educativa o nivel académico sobre el que se enfocaba; tampoco se indicaba la temática o asignatura curricular en la que se enmarcaba (p.e. "El aprendizaje cooperativo"; "El cuento en Educación Infantil"). En esta misma línea, los títulos en ocasiones no permitían discernir qué modalidad de TFG desarrollaban (revisión bibliográfica, investigación educativa o trabajo de innovación). Por otro lado, también observamos que en ocasiones se emplearon títulos más propios de unidades didácticas o programaciones (p.e. "Un arcoíris de emociones. Proyecto de innovación docente con propuestas didácticas sobre el fenómeno del pH”; “¡Nos vamos de excursión! Salidas escolares en Educación Primaria"). La presentación de trabajos con un título impreciso y/o poco representativo alcanzó el $62,8 \%$ de los casos aquí analizados (Tabla 2).

Tabla 1. Frecuencia de casos de los errores conceptuales detectados en la metodología de la investigación

\begin{tabular}{|c|c|}
\hline \multicolumn{2}{|c|}{ BLOQUE I. METODOLOGIA DE LA INVESTIGACIÓN } \\
\hline ITTEM SOBRE ERRORES DETECTADOS & $\begin{array}{l}\text { FRECUENCIA } \\
\text { DECASOS (\%) }\end{array}$ \\
\hline \multicolumn{2}{|l|}{ ESTRUCTURA Y ORGANIZACIÓN DEL TFG } \\
\hline Confunde modalidades de TFG & 41,9 \\
\hline $\begin{array}{l}\text { Introduce apartados que no se corresponden con los de } \\
\text { la modalidad elegida }\end{array}$ & 37,2 \\
\hline $\begin{array}{l}\text { Cree que un TFG es similar a una unidad didáctica o } \\
\text { programación }\end{array}$ & 34,9 \\
\hline $\begin{array}{l}\text { Cree que un TFG es similar a un proyecto educativo de } \\
\text { aula }\end{array}$ & 44,2 \\
\hline $\begin{array}{l}\text { Confunde los objetivos del TFG con los curriculares de } \\
\text { la etapa }\end{array}$ & 60,5 \\
\hline \multicolumn{2}{|l|}{ RECURSOS DE APOYO } \\
\hline No menciona en el texto las figuras y/o las tablas & 65,1 \\
\hline $\begin{array}{l}\text { No describe en el pie de figura y/o de tabla lo más } \\
\text { destacado de la ilustración }\end{array}$ & 58,1 \\
\hline $\begin{array}{l}\text { No indica la fuente de origen de los datos de las figuras } \\
\text { y/o tablas }\end{array}$ & 69,8 \\
\hline No menciona ni cita los anexos en el texto & 20,9 \\
\hline $\begin{array}{l}\text { Los anexos no presentan una breve descripción de su } \\
\text { contenido }\end{array}$ & 18,6 \\
\hline El uso de anexos está injustificado & 11,6 \\
\hline \multicolumn{2}{|l|}{ HIPÓTESIS } \\
\hline $\begin{array}{l}\text { La hipótesis formulada como pregunta o como objetivo } \\
\text { de investigación }\end{array}$ & 23,3 \\
\hline $\begin{array}{l}\text { La hipótesis formulada no puede ser contrastada con el } \\
\text { diseño presentado }\end{array}$ & 18,6 \\
\hline PROSPECTIVA & \\
\hline No se proponen temáticas para continuar el trabajo & 27,9 \\
\hline
\end{tabular}


Actualmente la existencia de publicaciones periódicas que permiten la activación de alertas a los usuarios suscritos y la digitalización de bases de datos accesibles a través de internet han permitido incrementar la visibilidad de los trabajos de investigación. Para facilitar esos procesos es importante elegir palabras clave definitorias del contenido. Nuestro estudio indicó que el $74,4 \%$ de los trabajos entregados contenían palabras clave que no ayudaban a precisar su contenido (Tabla 2). Ello demuestra que los alumnos tienen dificultades para elegir 5 palabras clave relevantes para cumplimentar este apartado.

Por último, la redacción cuidadosa del resumen juega un papel clave a la hora de despertar el interés del lector, proporcionándole una idea fidedigna y breve del contenido. Los estudiantes entregan un resumen con la idea que quieren desarrollar en la primera toma de contacto con el tutor, en él se describe lo que pretenden realizar en su TFG. Sin embargo, ese resumen se redacta como si el trabajo ya estuviese hecho, sacándose incluso conclusiones, a pesar de que no se ha iniciado la elaboración del mismo $(39,5 \%)$. Más llamativo resulta que tras entregar el primer borrador completo, habiendo obtenido resultados y conclusiones preliminares, el resumen siga siendo el mismo que se entregó antes de iniciar el trabajo, lo que ocurrió en el 34,9\% de los trabajos.

El tercer bloque de este estudio (Tabla 3) analizó los diferentes aspectos relacionados con la redacción del texto desde el punto de vista el contenido y la formalidad de la expresión escrita. En lo que respecta al contenido, hay dos ítems del cuestionario que lo abordan. El primero valora la conexión de la introducción con el resto del trabajo. La elaboración de una introducción bien estructurada que permita la conexión de la información previa con el resto de los apartados que va a tener el trabajo es fundamental para poner al lector en antecedentes y definir los aspectos relevantes que se van a analizar. Según nuestros datos, el $58,1 \%$ de los futuros maestros tienen dificultades para elaborar una introducción que conecte convincentemente con los resultados o acciones educativas que se plantean en su trabajo. Esta situación provoca que los contenidos más originales (resultados y discusión) queden desarticulados con respecto al marco teórico. Además, detectamos que en casi la mitad de los trabajos $(44,2 \%)$, el apartado de marco teórico carecía de la profundidad y detalles necesarios para mostrar al lector el ámbito en el que se centraba el estudio.

En cuanto a la formalidad de la redacción los alumnos mostraron una falta de formación en este sentido, ya que un $34,9 \%$ argumentaban usando la primera persona $\mathrm{y} / \mathrm{o}$ empleando opiniones personales sin base científica. Se manifiesta también una evidente falta de relación entre los objetivos propuestos en el estudio y las conclusiones alcanzadas $(46,5 \%)$. Esta situación puede tener relación con la concepción errónea que tienen los maestros de los objetivos de sus trabajos. Del mismo modo que las argumentaciones se producían en muchos casos sin base bibliográfica, en un $44,2 \%$ de los trabajos se detectaron niveles de plagio superiores a los permitidos según la guía docente $(<12 \%)$. Hay que indicar que algunos alumnos copiaban literalmente texto sin indicar la fuente, lo que estrictamente es plagio. Sin embargo, otros tenían graves dificultades para organizar la información de las fuentes y presentarla procesada con su correspondiente cita. En este caso se incluyen copia literal sin comillas y parafraseados incorrectos. Estos casos además han sido incluidos en el ítem 2 del bloque IV (Tabla 4). 
Tabla 2. Frecuencia de casos en los que se cometen errores conceptuales en los descriptores del trabajo.

\begin{tabular}{|c|c|}
\hline \multicolumn{2}{|l|}{ BLOQUE II. DESCRIPTORES } \\
\hline ÍTEM SOBRE ERRORES DETECTADOS & $\begin{array}{l}\text { FRECUENCIA } \\
\text { DE CASOS (\%) }\end{array}$ \\
\hline \multicolumn{2}{|l|}{ TÍTULO } \\
\hline $\begin{array}{l}\text { El título del TFG es poco representativo o } \\
\text { inadecuado }\end{array}$ & 62,8 \\
\hline \multicolumn{2}{|l|}{ PALABRAS CLAVE } \\
\hline Las palabras clave no perfilan el contenido del TFG & 74,4 \\
\hline \multicolumn{2}{|l|}{ RESUMEN } \\
\hline $\begin{array}{l}\text { Entregan un resumen completo antes de iniciar el } \\
\text { trabajo }\end{array}$ & 39,5 \\
\hline $\begin{array}{l}\text { El TFG conserva el mismo resumen que al inicio } \\
\text { del trabajo }\end{array}$ & 34,9 \\
\hline
\end{tabular}

Probablemente la discusión de un trabajo de investigación es la parte más difícil y la que requiere de mayor experiencia. Se trata de situar los nuevos datos aportados por el autor dentro del marco teórico y poner orden en la información previa, construyendo con espíritu crítico y reconfigurando un nuevo marco teórico para la comunidad científica. Los alumnos de magisterio no suelen estar habituados a realizar estas tareas y, posiblemente, por ello observamos que en esta faceta del trabajo se alcanzan altísimas frecuencias de trabajos con errores. El $60,5 \%$ de los alumnos no realizaron una discusión integradora de los datos presentados y los obtenidos de la bibliografía, así como tampoco una revisión crítica cuando la tipología del TFG lo requería. Un aspecto relacionado es el uso de citas para argumentar contenidos. Esto es la esencia de la discusión de los propios datos y de la construcción del nuevo conocimiento, pero en el $65,1 \%$ de trabajos no se utilizaron citas para apoyar las ideas nuevas. Quizás porque realmente no se construía una discusión crítica.

Del mismo modo no se abordaron los puntos débiles de la propuesta $(48,8 \%)$, cuya discusión suele ser el origen de la prospectiva, la cual no se incluyó en un tercio de los trabajos analizados.

Tabla 3. Frecuencia de casos en los que se cometen errores conceptuales en el formato de la redacción. 


\begin{tabular}{|c|c|}
\hline \multicolumn{2}{|c|}{ BLOQUE III. REDACCIÓN } \\
\hline ÍTEM SOBRE ERRORES DETECTADOS & $\begin{array}{c}\text { FRECUENCIA } \\
\text { DE CASOS } \\
\text { (\%) }\end{array}$ \\
\hline CONTENIDO & 44,2 \\
\hline Trata la temática sin profundidad & 58,1 \\
\hline $\begin{array}{c}\text { El marco teórico está desconectado del resto del } \\
\text { trabajo }\end{array}$ & 34,9 \\
\hline ESTILO DEL TEXTO & 46,5 \\
\hline $\begin{array}{c}\text { Argumenta usando la primera persona y empleando } \\
\text { opiniones personales sin base científica }\end{array}$ & 60,5 \\
\hline No concluye al hilo de los objetivos propuestos & 65,1 \\
\hline DISCUSIÓN & 48,8 \\
\hline $\begin{array}{c}\text { No hay discusión, revisión crítica ni análisis de los } \\
\text { contenidos }\end{array}$ & 44,2 \\
\hline Las argumentaciones no se justifican con citas \\
\hline $\begin{array}{c}\text { No se señalan los puntos débiles del TFG que se } \\
\text { podrian abordar en el futuro }\end{array}$ \\
\hline Copia literal sin cita de otros autores (plagio) \\
\hline LEGISLACIÓN & 25,6 \\
\hline Abuso de textos legales & 18,6 \\
\hline Uso injustificado de textos legales
\end{tabular}

Sorprendentemente, los alumnos de magisterio utilizan textos legales con profusión, dotando al texto de un alto nivel de literalidad obligada que no puede considerarse plagio. Además, en algunos casos la discusión se elabora en torno a las leyes. A pesar de ello, hay que destacar que uno de cada cuatro TFG presentaba un excesivo uso de textos legales $(25,6 \%)$, empleando incluso varias páginas para citar reales decretos conocidos y accesibles e incluso sin relación con el tema del trabajo $(18,6 \%)$.

Por último, el bloque IV engloba el análisis de los aspectos relacionados con la bibliografía (Tabla 4). El formato de TFG en la UCJC requiere que las referencias sean mencionadas siguiendo un formato habitual en las Ciencias Sociales, el de la Asociación Americana de Psicología (en inglés APA). En este caso, las referencias citadas en el texto son listadas al final del documento por orden alfabético de los apellidos del primer autor. Según nuestros datos, los trabajos de fin de grado de educación presentaron en un $95,3 \%$ de los casos incorrecciones de diferente grado en este aspecto. Los errores más frecuentemente detectados fueron que no se listaban las referencias por orden alfabético, ya que en ocasiones se ordenaban por el nombre del autor y en otras por el apellido dentro de la misma lista de referencias. Del mismo modo se hacía caso omiso del patrón definido por el formato APA para las referencias. Ambas circunstancias no fueron excluyentes y aparecían todo tipo de errores combinados.

También se observaron otras situaciones erróneas importantes relacionadas con la bibliografía. El formato APA sólo requiere listar entre las referencias aquellas obras que hayan sido mencionadas explícitamente en el texto, lo que no ocurría siempre en los trabajos presentados. Cuantificamos que en un $81,4 \%$ de los casos, los alumnos usaban como referencias páginas web, blogs y otras fuentes que no se citaban en el texto, y que en ocasiones aparecían como un subapartado de las referencias titulado como webgrafía. Los vídeos y otros recursos educativos de interés para la argumentación o presentación del trabajo son perfectamente válidos. Sin embargo, no pueden ser recogidos al final sin adecuarse al formato de referencias establecido. De hecho, el formato APA en su sexta edición explica pormenorizadamente cómo se debe citar y 
referenciar al final del documento cada una de las fuentes utilizadas según su origen, lo que incluye webs, blogs, vídeos, artículos de prensa o informes técnicos, entre otros.

La inclusión de fuentes no mencionadas en el texto en el apartado de referencias no fue exclusiva para contenidos virtuales. También se detectaron libros o artículos de investigación educativa publicados en revistas y libros de actas. Tan frecuente fue la situación que tres de cada cuatro alumnos $(74,4 \%)$ incurrieron en este error. Sin embargo, es más llamativo que se mencione en el texto una fuente (cita) y luego no se incorpore a la lista de referencias, lo que ocurrió en un $72,1 \%$ de los casos.

Por otro lado, detectamos que en muchos casos las referencias presentadas no eran actuales. Mención especial merece un caso en el que tratando un tema de innovación educativa a través de recursos informáticos no se mencionaban referencias de los últimos diez años. Casos como este indican que las búsquedas de información fueron poco exhaustivas o, en el mejor de los casos, poco eficaces. Este problema debe considerarse grave por la elevada frecuencia con la que ocurre $(72,1 \%)$.

Por último, dentro del bloque IV se encuentra un subapartado de citas incorrectamente empleadas que engloba dos ítems. Por un lado, en el $51,2 \%$ de los trabajos analizados aparecen citas en el texto que suelen estar fuera de la posición apropiada, ubicándolas al final del párrafo como norma general, lo cual no siempre es correcto. Con la misma frecuencia $(51,2 \%)$ observamos que en los borradores de TFG entregados se copiaban literalmente grandes fragmentos de texto con su correspondiente cita de autor y año. Esta forma de citar se debería considerar incorrecta, al menos en los trabajos fin de estudios, aunque se pueda permitir en determinados textos de gran volumen y en los que se mencionen a autores de gran relevancia para un tema.

Tabla 4. Frecuencia de casos en los que se cometen errores conceptuales en la elaboración de la bibliografía.

\begin{tabular}{|c|c|}
\hline \multicolumn{2}{|c|}{ BLOQUE IV. BIBLIOGRAFÍA Y REFERENCIAS } \\
\hline ÍTEM SOBRE ERRORES DETECTADOS & $\begin{array}{c}\text { FRECUENCIA } \\
\text { DE CASOS } \\
(\%)\end{array}$ \\
\hline \multicolumn{2}{|l|}{ FORMATO DE REFERENCIAS } \\
\hline $\begin{array}{l}\text { 1. No se utiliza el formato definido en la normativa } \\
\text { (APA) en el TFG entregado }\end{array}$ & 95,3 \\
\hline \multicolumn{2}{|l|}{ REFERENCIAS } \\
\hline 2. Presenta un subapartado de webgrafía & 81,4 \\
\hline 3. Las citas no se listan en referencias & 72,1 \\
\hline 4. Las referencias no se citan en el texto & 74,2 \\
\hline 5. Referencias desactualizadas & 72,1 \\
\hline \multicolumn{2}{|l|}{ CITAS } \\
\hline 6. Copia literal con comillas & 51,2 \\
\hline 7. Posición de la cita incorrecta & 51,2 \\
\hline
\end{tabular}




\section{Discusión y conclusiones}

Los resultados de nuestro estudio indican que los alumnos de magisterio cometen importantes fallos en la elaboración del Trabajo de Fin de Grado en el primer borrador del documento completo. Por supuesto que estos errores se subsanan antes de ser autorizados a defensa, y alcanzan el nivel exigido. Sin embargo, sería mejor que se pudiese intervenir sobre estas ideas antes y durante la elaboración del escrito y no al final del proceso de corrección de forma individual.

Con estos datos se puede decir que los maestros en formación tienen dificultades para entender qué es y cómo se hace la investigación educativa incluso mientras la están realizando. Esto se debe a que, entre los maestros en formación, pero también entre maestros en ejercicio, se cuestiona la utilidad real de la investigación educativa debido al enfoque que presenta (Perines y Murillo, 2017). En general los estudiantes de magisterio entienden que la forma de comunicar sus conocimientos a la comunidad educativa se establece a través de documentos como las programaciones y unidades didácticas. Sin embargo, esto no es real. La educación actual exige de procesos de intercambio de propuestas educativas innovadoras a través de otros foros como las revistas de investigación e innovación educativas. Por ello el lenguaje y el formato que se emplea en ellas deben ser conocidos y dominados por los futuros maestros como parte de su profesión, residiendo ahí la importancia y el objeto del TFG.

Esta nueva necesidad de los maestros pone en evidencia la formación en este sentido, ya que con los resultados obtenidos en nuestro estudio (TFG en fase de borrador completo) muchos de los estudiantes no distinguen la modalidad que entregan (revisión bibliográfica, investigación o innovación educativa). De este modo es evidente la relación que existe con otros resultados que mostramos, ya que en ocasiones tampoco presentan una adecuada hipótesis de trabajo y confunden los objetivos del aula con los del trabajo que realizan e incluso proponen únicamente programaciones didácticas como TFG. Tal situación de confusión entre la parte didáctica e investigadora ha sido descrita por otros autores. Gómez Barreto (2015) describe en su estudio que los TFG de magisterio presentan diversas carencias a nivel investigador, pero manifiestan un buen dominio didáctico de las situaciones. En esta línea, otros trabajos indican que la coincidencia temporal de Prácticum y TFG dificultan la asimilación correcta de las características propias de cada asignatura, provocando que predominen las acciones didácticas frente a las investigadoras (Cano Vela y Ortiz Ballesteros, 2013). Esta tendencia de distanciamiento respecto de la investigación tiene reflejo en otros trabajos que en ocasiones se solicitan a este tipo de alumnado. Tal es el caso del estudio de Egido et al. (2006) que, mucho antes de la implantación del TFG, desarrolló actividades formativas mediante aprendizaje basado en problemas. El análisis de los trabajos entregados indicaba que los estudiantes de magisterio presentan graves dificultades en la proposición de hipótesis. Este hecho está asociado con la infrecuencia de este tipo de trabajos a lo largo de sus estudios universitarios. Por todo ello, no se desarrolla la capacidad de visualización y análisis conjunto de toda la labor de elaboración del TFG, lo que probablemente impide a los estudiantes ver el recorrido intelectual que han hecho durante su trabajo y proponer una prospectiva de éste. Todo ello demuestra que no se capta completamente la esencia de lo que es la investigación educativa y la falta de dominio sobre la crítica y la argumentación, lo que también se puede comprobar en evaluaciones de otras asignaturas (Martí, Amat y Jiménez Bargalló, 2013).

Como muestran nuestros resultados, los alumnos de educación tienen serias dificultades para organizar la información y proponer ideas propias elaboradas a partir de la bibliografía obtenida, argumentar y discutir. Sin olvidar la forma en la que hacen mención a otros autores, cayendo en el plagio y/o en la presentación de un apartado de 
referencias mal elaborado. Estas dificultades de los alumnos en la búsqueda y gestión bibliográfica, así como en el análisis crítico, han sido descritas recientemente mediante estudios basados en las respuestas de los estudiantes a encuestas (Gómez Barreto, 2015; Faya, Guadamillas y Alcaraz, 2017). También observamos que muestran debilidades formativas en la forma de integrar las figuras y los anexos en el texto. Todos estos errores pueden tener origen en la infrecuencia de redactar trabajos en los que se pida esta organización, construcción y análisis crítico de la información, ya que en fuentes más cotidianas para los maestros (unidades didácticas) no suelen citarse en el texto ni las figuras ni las referencias, y tampoco se emplea un formato tan preciso como APA.

Los maestros también tienen dificultades a la hora de proponer títulos y palabras clave que describan claramente los contenidos de los que trata el trabajo. Las características que recomiendan para un título son muchas: brevedad, contundencia, que sea sugerente, provocador, enigmático, etc. (de la Ossa, 2016), pero siempre guardando unas formas de estilo académico que permitan relacionarlo con el tema que trata. Ésta es una tarea fundamental, ya que estos ítems sintetizan la esencia del TFG. Por ello, si no son capaces de elaborarlas en su propio trabajo, difícilmente podrán proponerlas para realizar búsquedas de información. Estos resultados complementan la idea de que no manejan correctamente las bases de datos de bibliografía (Faya et al., 2017), lo que tiene reflejo en el uso y presentación de la bibliografía. Por lo tanto, parece evidente que el principal problema y más extendido es la falta de capacidad de buscar la bibliografía. Si trabajásemos este aspecto, se activaría la búsqueda de respuestas y la integración de la información.

\section{Propuestas de mejora}

En nuestra opinión, quizás la solución pase por llevar a cabo acciones formativas (cursos y talleres) de búsqueda bibliográfica, lectura y análisis de artículos de investigación educativa, y a su vez deberían ser potenciadas en diferentes asignaturas a lo largo de la titulación, donde se soliciten la entrega de trabajos con este formato, de forma que se entienda esta labor como una circunstancia normal y no exclusiva de los TFG. Con ello, los alumnos aprenderán a manejar y presentar la bibliografía de forma adecuada, incluso mediante gestores bibliográficos. También interiorizarán la organización y estructura de los trabajos de investigación educativa, su utilidad en el aula y su trascendencia en la mejora educativa, de manera que podamos involucrarlos en esta nueva función de los docentes del siglo XXI.

En conjunto mejorarían los aspectos previos sobre el TFG, ayudando a entender el TFG como un formato en la transmisión y elaboración de la información en investigación educativa y sus diferentes modalidades. Por último, instamos a los tutores de TFG de los grados de Educación a comunicar sus experiencias respecto de las necesidades de mejora concretas y debilidades formativas de sus estudiantes de TFG para diseñar estrategias definidas de intervención en su aprendizaje antes de la elaboración del documento.

\section{Referencias}

Álvarez, M. y Pascual, M. (2012). Propuesta de evaluación del Trabajo Fin de Grado en Derecho. Aula Abierta, 40(1), 85-102.

Arteaga, B., Ahedo, J., Gutiérrez Gómez-Calcerrada, S. y Solera, E. (2013). El Trabajo Fin de Grado Maestro: modalidad online con evaluación presencial. RED. Revista de Educación a Distancia, 38. 
Bonilla, MJ., Fuentes, L., Vacas, C. y Vacas, T. (2012). Análisis del proceso de evaluación del Trabajo Fin de Grado en las nuevas titulaciones. Educade: revista de educación en contabilidad, finanzas y administración de empresas, 3, 5-21.

Cano Vela, AG. y Ortiz Ballesteros, MA. (2013). Prácticum y tfg: su imbricación en la formación de maestros. (1-11). RUIdeRA: Repositorio institucional de la Universidad de Castilla-La Mancha.

De la Ossa, MA. (2016). Una aproximación a la investigación para y desde el magisterio: orientaciones para superar el TFG y no morir en el intento. (1-29). Facultad de Educación de Cuenca: Universidad de Castilla-La Mancha.

Egido, I., Aranda, R., Cerrillo, R., De la Herrán, A., De Miguel, S., Gómez, M., Hernández, R., Izuzquiza, D., Murillo y FJ., Pérez, M. (2006). Aprendizaje basado en problemas (ABP). Estrategia metodológica y organizativa del currículum para la calidad de la enseñanza en los estudios de Magisterio. Revista Interuniversitaria de Formación del Profesorado, 20(3), 137-149

Espacio Europeo de Educación Superior (1999). Declaración de Bolonia. Ministerio de Educación Cultura y Deporte. Madrid. España.

Faya Cerqueiro, F., Guadamillas Gómez, MV. y Alcaraz Mármol, G. (2017). Limitaciones y conocimientos previos a la redacción del TFG: estudio de caso. En Ana María Martín Cuadrado, Esther Juan Oliva, y Nuria Carriedo López (Coords.), VIII Jornadas de Redes de Investigación en Innovación de la UNED. Los trabajos fin de carrera (TFG y TFM): el camino de la profesionalización. (539-544). Madrid: Editorial UNED.

Garrote, M. (2015). EI TFG: sus modalidades, objetivos y competencias a validar. Reflexiones a partir de la experiencia en la Facultad de Derecho de la UCM. Docencia y Derecho. Revista para la docencia jurídica universitaria, 9.

Gómez Barreto, MI. (2015). La evaluación del trabajo final de grado en las titulaciones de Maestro de Educación Infantil y Primaria de la Facultad de Educación de Albacete en la Universidad Castilla La Mancha. Opción, 31(3), 541-563.

Hernández-Leo, D., Moreno Oliver, V., Camps, I., Clarisó, R., Martínez-Monés, A., Marco-Galindo, MJ. y Melero, J. (2013). Implementación de buenas prácticas en los Trabajos Fin de Grado. Revista de Docencia Universitaria, 11, 259-278.

López-Cózar, C., Priede, T. y Benito Hernández, S. (2013). Análisis de la expresión escrita en las Universidades de Madrid a través de la asignatura Trabajo Fin de Grado en los estudios de ADE. Revista de Docencia Universitaria, 11(3), 279-299.

Martí, J., Amat, A. y Jiménez Bargalló, I. (2013). El uso de evidencias en la escritura científica en los estudiantes de magisterio. Textos de Didáctica de la Lengua y de la Literatura, 64, 40-46.

Mateo, J. (2009). Guía para la evaluación de competencias en el trabajo de fin de grado en el ámbito de las ciencias sociales y jurídicas. Barcelona: Agència per a la Qualitat del Sistema Universitari de Catalunya.

MEC (2007). RD 1393/2007, de 29 de octubre, por el que se establece la ordenación de las enseñanzas universitarias oficiales. BOE núm. 260,30 octubre.

MEC (2010). RD 861/2010, de 2 de julio, por el que se modifica el Real Decreto 1393/2007, de 29 de octubre, por el que se establece la ordenación de las enseñanzas universitarias oficiales. BOE 3 de julio de 2010.

Muñoz-Cantero, J. y Mato-Vásquez, MD. (2014). El proyecto docente en la universidad española según el Espacio Europeo de Educación Superior. Calidad en la educación, 40, 319-334.

Neave, G. (2003). The Bologna declaration: Some of the historic dilemmas posed by the reconstruction of the community in Europe's systems of higher education. Educational Policy, 17(1), 141-164.

Perines, H. y Murillo, FJ. (2017). ¿Cómo mejorar la investigación educativa? Sugerencias de los docentes. Revista de la Educación Superior, 46(181). 
Proyecto Tuning (2009). Una introducción a Tuning Educational Structures in Europe. Bilbao: Universidad de Deusto.

Rekalde Rodríguez I. (2011). ¿Cómo afrontar el trabajo fin de grado? Un problema o una oportunidad para culminar con el desarrollo de las competencias. Revista Complutense de Educación, 22(2), 179-193.

Reynolds, JA. y Thompson, RJ. Jr. (2011). Want to Improve Undergraduate Thesis Writing? Engage Students and Their Faculty Readers in Scientific Peer Review. CBE-Life Sciences Education, 10(2), 209215.

Rowley, J. y Slack, F. (2004). What is the future for undergraduate dissertations? Education + Training, 46(4), 176-181.

Rullan, M., Estapé-Dubreuil, G., Fernández, M. y Márquez, MD. (2010). La evaluación de competencias transversales en la materia Trabajo Fin de Grado. Un estudio preliminar sobre la necesidad y oportunidad de establecer medios e instrumentos por ramas de conocimiento. Revista de Docencia Universitaria, 8(1), 74-100.

Valderrama, E., Rullán, M., Sánchez, F., Pons, J., Mans, C., Giné, F., Seco, G., Jiménez, L., Peig, E., Carrera, J., Moreno, A., García, J., Pérez, J., Vilanova, R., Cores, F., Renal, JM., Tejero, J. y Bisbal, J. (2010). La Evaluación de Competencias en los Trabajos Fin de Estudios. IEEE-RITA, Revista Iberoamericana de Tecnologías del Aprendizaje, 5(3), 107-114.

Vera, J. y Briones, E. (2016). Un nuevo reto para las universidades: la evaluación de la calidad de la gestión de los Trabajos de Fin de Grado. Foro de Educación, 14(21), 281-310.

Webster, F., Pepper, D. y Jenkins, A. (2000). Assessing the undergraduate dissertation. Assessment \& Evaluation in Higher Education, 25(1), 71-80. 